\title{
Drug-facilitated crime caused by drinks or foods
}

\author{
Fatemeh Gharedaghi ${ }^{1}$, Hossein Hassanian-Moghaddam ${ }^{2,3^{*}}$ (D), Maryam Akhgari ${ }^{4}$, Nasim Zamani ${ }^{2,3}$ \\ and Fakhreddin Taghadosinejad ${ }^{5}$
}

\begin{abstract}
Background: Offenders performing drug-facilitated crime (DFC) generally choose their drug based on its availability and their own attitude. Prevalence of DFC has increased in our society triggering some public concerns. Treatment of the patients strongly depends on the abused drug while the type of the drug involved is generally unknown; hence, we performed a study to determine the most frequently abused drugs to facilitate crimes in Iran.

Methods: In a prospective study, all patients who were referred to our medical toxicology ward due to DFC between February 2009 and March 2010 were included. A self-made questionnaire was filled out for each patient. Urine samples were taken on presentation, and drugs were extracted by organic solvents using liquid-liquid extraction (LLE) method. Forensic toxicology urine analyses were performed using immunochromatography and thin layer chromatography (TLC) as screening tests. High-performance liquid chromatography (HPLC) and gas chromatography/mass spectrometry (GC/MS) were used as confirmatory methods.
\end{abstract}

Results: In 53 patients, 8 (15.1\%) were female and the mean age was $31.6 \pm 12.5$ years. They were mainly poisoned to facilitate theft (77.4\%). Drugs were commonly given in drinks (45.3\%) and during transportations (45.4\%). The patients had usually been referred with mild loss of consciousness (56.6\%), and the most common drugs given were metoclopramide and dextromethorphan (18.9\%). No death was documented, and the mean hospitalization period was 1 day.

Conclusion: Opioids are the most commonly involved drugs in DFCs in Iran. Metoclopramide, benzodiazepines, and antihistamines are other common drugs used for this purpose. Public health education, training, and empowerment of medical staff dealing with investigating and management of DFC are required.

Keywords: Drug-facilitated crime, Poisoning, Forensic toxicology analysis, Sedative-hypnotics, Opioids

\section{Background}

Drug-facilitated crimes (DFCs) have globally increased and are currently a socio-medical emergency worldwide (Zerbo et al. 2018). Drug-facilitated crimes are defined as crimes such as robbery, sexual assault, money extortion or battery when committed while the victim is under the influence of drugs (Wang et al. 2018). Victims are generally people who accept food and drinks offered by strangers during the intra-city journeys or at work and are referred to emergency departments by emergency medical services

\footnotetext{
* Correspondence: hassanian@sbmu.ac.ir

${ }^{2}$ Social Determiniants of Health Research Center, Shahid Beheshti University of Medical Sciences, Koodakyar Ave., Daneshju Blvd., Velenjak, Tehran, Iran

${ }^{3}$ Department of Clinical Toxicology, Shahid Beheshti University of Medical Sciences, Tehran, Iran

Full list of author information is available at the end of the article
}

(EMS) or by their friends/colleagues. Their treatment protocol is usually conservative.

Benzodiazepines are among the most frequently used substances in DFCs due to their strong numbing effect. They can be easily obtained from pharmacies for criminal intents (Piergiovanni et al. 2018). According to the previous studies, lorazepam, diazepam, nitrazepam, flunitrazepam, clonazepam, bromazepam, non-benzodiazepine sedatives, zopiclone, and zolpidem are the most commonly used drugs in DFCs (Wang et al. 2018; Piergiovanni et al. 2018). Since flunitrazepam-induced anterograde amnesia is severe, it is generally favored by the offenders (Grela et al. 2018).

There are reports that drug-laced beverages are made by the unsolicited addition of drugs to food and beverages that are linked to drug-facilitated crimes such as rape 
(Chan and Ramli 2018). Incapacitating ethanol or drugs (medicinal/recreational) are among substances that are used for drug-facilitated crimes (Tiemensma and Davies 2018). Ethanol, street drugs (including hashish and cocaine), and fast-acting benzodiazepines, as well as GHB have also been used for this purpose because they affect fast and cause muscle relaxation and anterograde amnesia (Milhorn 2018). Alcohol use is accompanied by cognitive impairment that results in diminishing acuity. High blood alcohol levels can render an individual unconscious and incapable (Anderson et al. 2017).

In forensic toxicology, liquid chromatography/mass spectrometry (LC/MS) is often used to detect trace amounts of benzodiazepines after their ingestion. However, some drugs are rapidly eliminated from the body or the patients refer very late, yielding negative results.

Diagnosis of drug-facilitated rape is difficult due to multiple positive drug results in the patients' samples (Grela et al. 2018). The real number of the rapes facilitated by drugs is more than that reported because many of the victims postpone reporting the crime for several days. Performance of confirmatory tests is also difficult because these drugs affect in low doses and are chemically unstable and metabolized, and excreted from the body very fast.

The issue of DFC is a hidden, serious public health and legal problem in Iran. The aim of the present study was to identify the most common medications used for DFC in Tehran, Iran. To the best of our knowledge, no similar study has been performed to systematically evaluate DFCs in Iran.

\section{Methods}

In a prospective observational study between February 2009 and March 2010, all patients who were referred to two university hospitals of Tehran with the chief complaint of being poisoned by another person to facilitate a crime (DFCs) were included. If the patients had referred with loss of consciousness (LOC), they were included after regaining consciousness and giving history. All urine samples were taken on presentation. A self-made questionnaire containing information on the victims' age and gender, their occupation, level of education, place of poisoning, food or drink ingested by the victim, accompanying person to the health care facility, on-arrival vital signs and level of consciousness, offender's age and gender (if known), history of acquaintance with the offender, motive for crime (if known), and type of the drug used for this purpose was filled out for every single patient (Additional file 1).

Collection, transfer, and storage of the biological samples were performed according to the United Nations Office on Drugs and Crime (UNODC) protocol (Guidelines for the forensic analysis of drugs facilitating sexual assault and other criminal acts 2011). On admission, a 30-mL urine as well as a $10-\mathrm{mL}$ whole blood sample was collected with patient's or his/her guardian/accompanying person's permission. According to the lab protocol, all urine samples were checked for adulteration by estimation of the specific gravity and $\mathrm{pH}$. Urine and blood samples were kept in plastic containers in $+4{ }^{\circ} \mathrm{C}$ refrigerators. All samples were transferred to forensic toxicology laboratories in Legal Medicine Organization, Tehran, Iran for toxicological analysis. Patients who did not wish to give urine samples and those who mentioned consumption of any medication or drug in the week prior to admission that could interfere with laboratory results were considered to be excluded. If the patients had a positive addiction history or used over-the-counter (OTC) or prescribed drugs, they were only included in case they had positive results for drugs other than the drug they had used.

For toxicological analysis, urine samples were extracted by liquid-liquid extraction (LLE) in systematic toxicological analysis (STA) manner for screening drugs, poisons, and opioids (Kordrostami et al. 2017). As novel psychoactive substances are not available and routinely used in Iran, we did not search for them in biological specimens. All urine samples were tested for amphetamine-type stimulants (ATS) (cut-offs for amphetamine and methamphetamine were $500 \mathrm{ng} / \mathrm{mL}$ ), opioids (cut-offs for morphine and codeine were $300 \mathrm{ng} / \mathrm{mL}$ ), methadone (cut-off $=300 \mathrm{ng} / \mathrm{mL})$, tramadol $\quad$ (cut-off $=200 \mathrm{ng} / \mathrm{mL}$ ), benzodiazepines (cut-off $=300 \mathrm{ng} / \mathrm{mL}$ ), Delta (9)-tetrahydrocannabinol $(\mathrm{THC})$ (cut-off $=50 \mathrm{ng} / \mathrm{mL})$, cocaine (cut-off $=300 \mathrm{ng} / \mathrm{mL}$ ), tricyclic antidepressants (TCA) $(1000 \mathrm{ng} / \mathrm{mL}$ ), barbiturates (cut-off $=300 \mathrm{ng} / \mathrm{mL}$ ), and phencyclidine $(\mathrm{PCP}) \quad($ cutoff $=25 \mathrm{ng} / \mathrm{mL})$ using immunochromatography tests.

For the specific analysis of ATS, heptafluorobutiric acid (HFBA) was used as derivatization reagent. The $\mathrm{pH}$ of $2 \mathrm{~mL}$ urine sample was adjusted to 12, and the desired analytes (amphetamine and methamphetamine) were extracted using n-hexane $(3 \mathrm{~mL})$ and shaking for $20 \mathrm{~min}$. Organic layer was separated by immersion of the sample in freezing bath. For derivatization step, $25 \mu \mathrm{L}$ of HFBA was added to the medium and vortexed for $3 \mathrm{~min}$. Organic layer was separated in crimp-capped vials and analyzed using GC/MS instrumentation (Bahmanabadi et al. 2017).

Urine samples were hydrolyzed using hydrochloric acid and heating for $60 \mathrm{~min}$ on $90^{\circ} \mathrm{C}$ water bath. As many drugs of forensic interest have alkaline chemical structure, the $\mathrm{pH}$ of the urine sample was adjusted to basic $(\mathrm{pH}=12)$ for efficient extraction of alkaline drugs such as narcotic analgesics, benzodiazepines, and ATS. Careful adjustment of $\mathrm{pH}$ to 8.5-9 was needed for the extraction of amphoteric drugs such as morphine using LLE method (Kordrostami et al. 2017). For the extraction of acidic drugs such as phenytoin, barbiturates, and pyrimidine, 
the $\mathrm{pH}$ of experiment medium was adjusted to 2 . After $\mathrm{pH}$ adjustment, $2 \mathrm{~mL}$ of urine sample was extracted using three aliquots of $2 \mathrm{~mL}$ chloroform: isopropanol $(8: 2, v / v)$. The mixture was vortexed for $10 \mathrm{~min}$ and centrifuged for $15 \mathrm{~min}$ at $6000 \mathrm{rpm}$. The organic layer was separated and evaporated to dryness under gentle nitrogen stream gas. Residues were analyzed using TLC method as screening and high-performance liquid chromatography (HPLC) and gas chromatography/mass spectrometry (GC/MS) instrumentation as confirmatory methods. Pre-coated TLC aluminum sheets $(20 \times 20)$, with $0.25 \mathrm{~mm}$ silica gel layer thickness and ultraviolet fluorescent indicator ALUGRAM ${ }^{\bullet}$ Xtra SIL G SIL UV254, MachereyNagel Gmbh, Duren, Germany, were used as screening method (Bazmi et al. 2016; Akhgari et al. 2018). It should be noted that all samples were searched for pregabalin and baclofen.

Primary positive results were confirmed using HPLC (Knauer, Germany) with a diode array detector (DAD; Knauer DAD 2700, Germany) plus a quaternary pump (Knauer pump 1000, Germany) and GC/MS (Agilent 7890A, USA) with a mass detector (5975C) to confirm positive results. Blood samples were analyzed for ethanol quantitatively using headspace gas chromatograph $6890 \mathrm{~N}$ (Agilent Technologies Inc.) and flame ionization detector (FID) (Behnoush et al. 2010).

All analytical methods were pre-validated in the lab and showed acceptable inter- and intra-assay precision. Limit of detection (LOD) and limit of quantitation (LOQ) were acquired for all analytes that are important from forensic toxicology point of view such as opioids, ATS, ethanol (LOD and LOQ were 0.1 and $1 \mathrm{mg} / \mathrm{dL}$ ), methadone, tramadol, benzodiazepines, barbiturates, and tricyclic antidepressants. .

It should be emphasized that our lab is routinely evaluated by internal and external (UNODC) quality control procedures twice a year. Our lab was accredited by UNODC from 2015.

The data was extracted into SPSS software version 16 and analyzed. For the description of quantitative variables with normal and non-normal distribution, mean $( \pm \mathrm{SD})$ and median (inter-quartile range) were used, respectively. For qualitative variables, percent of frequency was mentioned. To evaluate the association between categorical variables, chi-square test was used. Mann-Whitney $U$ test was used to compare differences between two independent groups when the dependent variable was continuous, but not normally distributed. A $p$ value less than 0.05 was considered to be statistically significant.

\section{Results}

A total of 53 patients were prospectively evaluated, of whom, $45(84.9 \%)$ were male and all were Iranians. Their mean age was $31.6 \pm 12.4$ years (mean \pm SD) with an age range of 7 to 65 years. Basic and demographic characteristics of the cases are shown in Table 1.

Almost $55 \%$ of the victims mentioned no change in the taste of their food/drink. Medications were given by the offenders in a fraud action to examine the victims' health status to offer them a job if they were determined to be healthy, or as a vitamin supplement, and/or to threaten them. The patients had mainly referred with mild LOC (Glasgow Coma Scale $[\mathrm{GCS}]=14 ; 56.6 \%$ ) or completely awake $(\mathrm{GCS}=15 ; 26.4 \%)$ although in most of them LOC had improved during transferring to hospital. Two car accidents had happened in driver victims with no major trauma.

The offenders were male in almost $78 \%$, female in $5.7 \%$, and both in $11.3 \%$ of the cases and about $75 \%$ of them were strangers. They offered something to eat (71\%), gave free food on religious ceremonies (13.2\%), and offered medication for treatment of a medical situation (such as headache) or as a part of employment process (in $15.1 \%$ of the cases). The offenders' estimated range of age was 20 to 50 years although three victims had no idea about or had forgotten the offender's approximate age. In 20 victims (37.7\%), the offender had pretended to eat or drink the food/juice.

In 49 cases (92.5\%), no history of recent drug/medication use was present. One case claimed to be on addiction withdrawal protocol using codeine and ibuprofen, one used tramadol, one was on diabetic and cardiovascular medications, and one mentioned infrequent use of amphetamine. Although we searched for ATS, cocaine, pregabalin, and baclofen in urine samples, none of the cases were positive for any of them. Forty-seven (88.7\%) and 4 (7.5\%) victims were hospitalized for 1 day or less and 2 days, respectively, while 2 were not hospitalized at all. No death was reported. Median time between exposure and urine collection was $11 \mathrm{~h}$ (range 1 to $120 \mathrm{~h}$ ). On-arrival vital signs were normal in all cases except one.

The detected drugs are shown in Table 2. All of the detected drugs were above cut-off levels. Metoclopramide was positive in $10(18.9 \%)$ cases, 5 of whom were poisoned in the work place $(P=0.001)$. Metoclopramide was less used when the offender tasted the food him/herself to gain the victim's trust $(P=0.008)$. Type of the drug and period of hospitalization were not significantly correlated. Patients significantly referred when they had sensed taste change in their food/drink ( $7 \mathrm{~h}[3,12$; range 1 to 120$]$ versus $2 \mathrm{~h}$ [1.5, 6 ; range 1 to 48]; $P=0.004$ ). Type of the drug was neither related to the motive of administration nor to the offender's gender.

\section{Discussion}

No statistics exist on the criminal poisonings in Iran. However, it seems that the number of these poisonings has increased during the recent years. This may be due 
Table 1 Demographic and basic characteristics of the drugfacilitated crime victims

\begin{tabular}{|c|c|}
\hline Variable & Results \\
\hline Mean ( \pm SD) age year (range) & $31.6 \pm 12.4(7-65)$ \\
\hline \multicolumn{2}{|l|}{ Sex, $n(\%)$} \\
\hline Female & $8(15.1)$ \\
\hline Male & $45(84.9)$ \\
\hline \multicolumn{2}{|l|}{ Schooling year ${ }^{\mathrm{a}}, n(\%)$} \\
\hline 0 & $5(9.4)$ \\
\hline $1-5$ & $9(16.9)$ \\
\hline $6-8$ & $9(16.9)$ \\
\hline $9-12$ & $22(41.5)$ \\
\hline$>12$ & $7(13.2)$ \\
\hline \multicolumn{2}{|l|}{ Job, $n(\%)$} \\
\hline Worker & $22(41.5)$ \\
\hline Clerks & $7(13.2)$ \\
\hline Unemployed persons & $6(11.3)$ \\
\hline Housekeepers & $5(9.4)$ \\
\hline Sellers & $4(7.5)$ \\
\hline Scholars & $4(7.5)$ \\
\hline Drivers & $2(3.8)$ \\
\hline Other jobs & $3(5.7)$ \\
\hline \multicolumn{2}{|l|}{ Refer, $n(\%)$} \\
\hline EMS & $21(39.6)$ \\
\hline Friends & $15(28.3)$ \\
\hline Relatives & $12(22.6)$ \\
\hline \multicolumn{2}{|l|}{ Motive, $n(\%)$} \\
\hline Robbery & $41(77.4)$ \\
\hline Rape & $2(3.8)$ \\
\hline Not defined & $10(18.8)$ \\
\hline \multicolumn{2}{|l|}{$\begin{array}{l}\text { Location of crime, } \\
n(\%)\end{array}$} \\
\hline Intra-city travels & $10(18.9)$ \\
\hline Inter-city travels & $9(17)$ \\
\hline Terminals & $4(7.5)$ \\
\hline Workplaces & $10(18.8)$ \\
\hline Within the city & $10(18.8)$ \\
\hline Victim's property & $7(13.2)$ \\
\hline Party & $3(5.7)$ \\
\hline $\begin{array}{l}\text { Mean }( \pm S D) G S^{b} \\
\text { (range) }\end{array}$ & $\begin{array}{l}13.98 \pm 0.88 \\
(12-15)\end{array}$ \\
\hline \multicolumn{2}{|l|}{ Edible offered, $n$ (\%) } \\
\hline Juice & $20(37.7)$ \\
\hline Food & $7(13.2)$ \\
\hline Chocolate & $6(11.3)$ \\
\hline Medicine & $6(11.3)$ \\
\hline Biscuit & $5(9.4)$ \\
\hline
\end{tabular}

Table 1 Demographic and basic characteristics of the drugfacilitated crime victims (Continued)

\begin{tabular}{ll}
\hline Variable & Results \\
\hline Cake & $4(7.5)$ \\
Tea & $2(3.8)$ \\
Other drinkable liquids & $3(5.7)$ \\
Median $[\mathrm{IQR}]$ crime elapsed time (h) & $3[2,8](1-120)$ \\
Median $[\mathrm{IQR}]$ test elapsed time (h) (range) & $11[3.7,20.5](1,120)$ \\
Mean ( $\pm \mathrm{SD})$ hours of admission length (range) & $25 \pm 8(0-48)$ \\
\hline
\end{tabular}

${ }^{\mathrm{a}} \mathrm{A}$ missing data

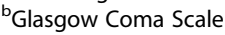

to the high availability of the drugs specially opioids and sedative-hypnotics (Hassanian-Moghaddam 2013; Hassanian-Moghaddam et al. 2014a; Soltaninejad et al. 2014; Hassanian-Moghaddam et al. 2014b; Piergiovanni et al. 2018). UNODC has previously published a guideline for the forensic analysis of drugs facilitating sexual assault and other criminal acts indicating that some drug categories including benzodiazepines, antihistamines, barbiturates, opioids, ATS, cannabis, and alcohols are identified to be used to perpetrate DFC (Guidelines for the forensic analysis of drugs facilitating sexual assault and other criminal acts 2011). Alcohol may be used as an incapacitating aid (Grela et al. 2018); however, in the

Table 2 Detected drugs in the urine samples of the drug-facilitated crime victims

\begin{tabular}{llll}
\hline Drug group & Drug name & $N(\%)$ & Instrumentation used \\
\hline Opioids & Dextromethorphan & $10(18.5)$ & GC/MS \\
& Tramadol & $5(9.2)$ & HPLC, GC/MS \\
& Methadone & $3(5.5)$ & GC/MS \\
& Codeine & $3(5.5)$ & HPLC, GC/MS \\
& Levorphanol & $1(1.9)$ & GC/MS \\
& Morphine & $1(1.9)$ & GC/MS \\
Antihistamines & Promethazine & $2(3.7)$ & HPLC, GC/MS \\
& Cyproheptadine & $1(1.9)$ & HPLC \\
Benzodiazepines & Lorazepam & $4(7.4)$ & HPLC \\
& Nortetrazepam & $3(5.5)$ & HPLC \\
Other drugs & Metoclopramide & $10(18.5)$ & GC/MS \\
& Theobromine & $5(9.2)$ & GC/MS \\
& Phenobarbital & $1(1.9)$ & GC/MS \\
& Amitryptyline & $1(1.9)$ & HPLC, GC/MS \\
& Imipramine & $1(1.9)$ & HPLC, GC/MS \\
& Clomipramine & $1(1.9)$ & HPLC, GC/MS \\
& Carbamazepine & $1(1.9)$ & HPLC \\
& Propranolol & $1(1.9)$ & HPLC \\
\hline
\end{tabular}


Khan's study-with cultural similarities to us-ethanol was not a preferred option for DFCs (Khan et al. 2014).

Higher frequency of poisoning in males shows the fact that young males are more actively present in the society and carry more money. Erfan Uddin and colleagues reported that mean age of the victims was 31.23 years with a predominant male tendency (100\%) (Erfan Uddin et al. 2018). Other studies showed the higher frequency of poisoning in males between 25 and 55 years (Khan et al. 2014).

Almost $50 \%$ of the victims were undergraduate (lower than diploma) suggesting that higher educational levels might be a protective factor for such poisonings. Approximately, $42 \%$ of the victims were workers, a finding that was previously shown by similar studies (Khan et al. 2014; Kuwayama et al. 2018).

About $43 \%$ of our victims were intoxicated by poisonous drinks unwillingly (Grela et al. 2018; Kuwayama et al. 2018); a finding in accordance with the results of the previous studies. Almost $55 \%$ of the victims mentioned no taste change, and in case the victim had sensed the change in the taste of the drink, he/she had referred to health care facilities late. We could not explain the cause of this finding.

Most poisonings had happened during transportations (45.4\%). Erfan Uddin et al. defined travel-related poisonings, almost $55 \%$ of which had happened in public busses (Erfan Uddin et al. 2018). This type of poisoning has also been reported in other studies in the region (Khan et al. 2014). Almost $38 \%$ of the offenders pretended to eat from the same drink or food to gain the victims' trust. This trick had never been reported before.

Although most of our patients had GCS of 14 or 15 on presentation, other studies had shown deeper on presentation loss of consciousness (Erfan Uddin et al. 2018; Khan et al. 2014). This may be interpreted by longer elapsed time between crime and emergency department presentation. Almost $92 \%$ of the cases had no history of drug use/ abuse. One case claimed that he was on addiction abstinence protocol, and another victim abused hashish infrequently, both of whom had negative test results. Most of our patients had been hospitalized for only $24 \mathrm{~h}$, and no death was reported. Vital signs were normal in all patients except for a 46-year-old male. Length of hospitalization was less than 3 days in most studies.

Many studies showed that benzodiazepines were the main cause of toxicity in cases of robbery, while in our study, many other types of drugs had been used by the offenders (Erfan Uddin et al. 2018; Khan et al. 2014; Singh et al. 2015). In previous studies in the UK, diazepam, temazepam, ecstasy, and GHB were used for drug-facilitated sexual assault (Grela et al. 2018).

Dextromethorphan was positive in 10 cases (using $\mathrm{GC} / \mathrm{MS}$ ) mainly in the intra-city poisonings. In 10 cases, no drug was found in analysis, and in 11 cases, non-sedative drugs (such as metoclopramide) were positive. It should be emphasized that negative results in DFC victims does not necessarily mean that no drug has been administered. This may be due to the delay in reporting and drug metabolism/excretion from the body (Grela et al. 2018). Maybe, the negative test results for sedative-hypnotics were due to the delay in the secretion of the metabolite or the principle drug was rapidly eliminated from the body. The problem of short half-lives of benzodiazepines has already been discussed and using improved methods including liquid chromatography-tandem mass spectrometry (LC-MS/MS) or GC-MS/MS is recommended (Drummer et al. 2019; Remane et al. 2014).

Using opioids as the first choice drug in DFCs has never been discussed before (Khan et al. 2014; Singh et al. 2015; Piergiovanni et al. 2018). It seems that easy availability of opioids made them a suitable weapon for such organized crimes. Another theory is the long detection period of opioids compared to short-acting sedativehypnotics resulting in more positive results.

Using antihistamines is rarely discussed in the literature (Wang et al. 2018). Antihistamines can induce sedation and may have synergistic effects with other sedative hypnotics (Grela et al. 2018). Administration of metoclopramide in conjunction with other drugs had not been discussed earlier, and it may be administered to avoid nausea and vomiting induced by intoxication in the victims. Using antiepileptics is usually limited to barbiturates but carbamazepine was discussed as well (Dinis-Oliveira and Magalhães 2013).

The most important limitation of the current study is habitual and without prescription use of the drugs which is a common problem in general population. We did not measure the concentration of the medications in biological samples obtained from patients. This is another major limitation of this study. Since hair and nail analyses were not feasible, history of recent drug use was relied on and urine drugs were checked by GC/MS (excluded if the test results and history matched) which could yield distorted results. The other limitation was missing of the possible deceased patients because this study was only performed in hospitalized patients. Cultural situation of the society that does not let the victims claim their sexual assault is another limitation that may cause case missing. In most cases, blood sample was collected too late and drugs were distributed and metabolized in the body; therefore, we only could detect drugs and their metabolites in urine samples qualitatively. Also plasma, serum, or blood were not available enough or were present with low quality in some instances. Moreover, the presence of sedative hypnotic drugs in urine samples without previous medical complications indicates that victims had used the drug unintentionally and were under the influence of drugs. 


\section{Conclusion}

Opioids may be used in DFCs in Iran. Metoclopramide, benzodiazepines, and antihistamines are other common drugs used for this purpose. Although we have no access to previous statistics on DFC, we estimate that its prevalence has increased. The ministry of health should definitely control OTC drug purchase. The media should warn the lay people about the possible dangers of accepting food or drinks from the strangers especially in terminals and public transportation facilities. Hair evaluation technology is needed to be set up in forensic and legal medicine laboratories in our country.

\section{Additional file}

Additional file 1: Drug-Facilitated Crime Questionnaire. (DOCX $16 \mathrm{~kb}$ )

\begin{abstract}
Abbreviations
DAD: Diode array detector; DFC: Drug-facilitated crime; EMS: Emergency medicine service; GC/MS: Chromatography/mass spectrometry; GCS: Glasgow Coma Scale; GHB: Gamma hydroxylbutyrate; HPLC: High performance liquid chromatography; HSGC: Head space gas chromatography; LC/MS: Liquid chromatography/mass spectrometry; LC/MS/MS: Liquid chromatography-tandem mass spectrometry; LLE: Liquid-liquid extraction; LOC: Loss of consciousness; OTC: Over-the-counter; SD: Standard deviation; STA: Systematic toxicological analysis; TLC: Thin layer chromatography; UNODC: United Nations Office on Drugs and Crime
\end{abstract}

\section{Acknowledgements}

None

\section{Funding}

None

\section{Availability of data and materials}

The datasets generated during and/or analyzed during the current study are available from the corresponding author on reasonable request.

\section{Authors' contributions}

The study was designed by HHM and FT. FG implemented the study. MA analyzed the biological samples. HHM analyzed the results. HHM and NZ drafted the manuscript, and all authors read and approved final draft

\section{Ethics approval and consent to participate}

Ethics approval was obtained from Shahid Beheshti University of Medical Sciences (IR.SBMU.RETECH.REC.1397.31)

\section{Consent for publication}

Not applicable

\section{Competing interests}

The authors declare that they have no competing interests.

\section{Publisher's Note}

Springer Nature remains neutral with regard to jurisdictional claims in published maps and institutional affiliations.

\footnotetext{
Author details

'Soorena Hospital, Tehran University of Medical Sciences, Tehran, Iran. ${ }^{2}$ Social Determiniants of Health Research Center, Shahid Beheshti University of Medical Sciences, Koodakyar Ave., Daneshju Blvd., Velenjak, Tehran, Iran. ${ }^{3}$ Department of Clinical Toxicology, Shahid Beheshti University of Medical Sciences, Tehran, Iran. ${ }^{4}$ Legal Medicine Research Center, Legal Medicine Organization (LMO), Tehran, Iran. ${ }^{5}$ Department of Forensic Medicine, Tehran University of Medical Sciences, Tehran, Iran.
}

Received: 28 August 2018 Accepted: 28 November 2018

Published online: 13 December 2018

\section{References}

Akhgari M, Amini-Shirazi N, Iravani FS (2018) Forensic toxicology perspectives of methadone-associated deaths in Tehran, Iran, a 7-year overview. Basic Clin Pharmacol Toxicol 122:436-441

Anderson LJ, Flynn A, Pilgrim JL (2017) A global epidemiological perspective on the toxicology of drug-facilitated sexual assault: a systematic review. J Forensic Legal Med 47:46-54

Bahmanabadi L, Akhgari M, Jokar F, Sadeghi HB (2017) Quantitative determination of methamphetamine in oral fluid by liquid-liquid extraction and gas chromatography/mass spectrometry. Hum Exp Toxicol 36:195-202

Bazmi E, Behnoush B, Akhgari M, Bahmanabadi L (2016) Quantitative analysis of benzodiazepines in vitreous humor by high-performance liquid chromatography. SAGE Open Med 4:2050312116666243

Behnoush B, Bazmi E, Akhgari M, Hashemi Nazari SS, Sardari Iravani F (2010) Evaluation of ethanol and N-propanol in victims. Iran J Toxicol 4:311-316

Chan KW, Ramli SH (2018) Development of an in-house HPLC method for the analysis of ecstasy-laced beverages. Egypt J Forensic Sci 8:18

Dinis-Oliveira RJ, Magalhães T (2013) Forensic toxicology in drug-facilitated sexual assault. Toxicol Mech Methods 23:471-478

Drummer OH, Di Rago M, Gerostamoulos D (2019) Analysis of benzodiazepines for drug-facilitated assaults and abuse settings (urine). In: Langman L, Snozek C (eds) LC-MS in drug analysis. Methods in Molecular Biology. Humana Press, New York, p 1872

Erfan Uddin RAMD, Ghose A, Uddin Hassan MDM, Kuch U, Kumar Dutta A, Zahed ASMD, Jabed SM (2018) A Clinico-epidemiological study on poisoning among commuters: is there any substance abuse? Asia Pac J Med Toxicol 7:38-41

Grela A, Gautam L, Cole MD (2018) A multifactorial critical appraisal of substances found in drug facilitated sexual assault cases. Forensic Sci Int 292:50-60

Guidelines for the forensic analysis of drugs facilitating sexual assault and other criminal acts (2011) Laboratory and Scientific Section United Nations Office on Drugs and Crime, Vienna https://www.unodc.org/documents/scientific/ forensic analys of drugs facilitating_sexual_assault_and other_criminal acts.pdf. Accessed 12 Sept 2017.

Hassanian-Moghaddam H (2013) An educational and research opportunity for the largest university hospital poison control centers; Tehran and Cairo. Egypt J Forensic Sci 3:64-65

Hassanian-Moghaddam H, Noroozi A, Zafaghandi S, Bagher M, Sarjami S (2014a) Substance abuse warning network: Pilot results in poisoned patients. Razi J Med Sci 21:47-58 [in Persian]

Hassanian-Moghaddam H, Zamani N, Rahimi M, Shadnia S, Pajoumand A, Sarjami S (2014b) Acute adult and adolescent poisoning in Tehran, Iran; the epidemiologic trend between 2006 and 2011. Arch Iran Med 17:534-538

Khan TM, Mehr MT, Ullah H, Abrar A (2014) Drug-facilitated street and travel related crime: a new public health issue. Gomal J Med Sci 12:205-209

Kordrostami R, Akhgari M, Ameri M, Ghadipasha M, Aghakhani K (2017) Forensic toxicology analysis of self-poisoning suicidal deaths in Tehran, Iran; trends between 2011-2015. Daru 25:15

Kuwayama K, Nariai M, Miyaguchi H, Iwata YT, Kanamori T, Tsujikawa K, Yamamuro T, Segawa H, Abe H, Iwase H, Inoue H (2018) Micro-segmental hair analysis for proving drug-facilitated crimes: evidence that a victim ingested a sleeping aid, diphenhydramine, on a specific day. Forensic Sci Int 288:23-28

Milhorn HT (2018) Sedative-hypnotic dependence. In: Substance Use Disorders. Springer, Cham, pp 59-76

Piergiovanni M, Cappiello A, Famiglini G, Termopoli V, Palma P (2018) Determination of benzodiazepines in beverages using green extraction methods and capillary HPLC-UV detection. J Pharm Biomed Anal 154:492-500

Remane D, Wetzel D, Peters FT (2014) Development and validation of a liquid chromatography-tandem mass spectrometry (LC-MS/MS) procedure for screening of urine specimens for 100 analytes relevant in drug-facilitated crime (DFC). Anal Bioanal Chem 406:4411-4424

Singh SP, Kaur S, Singh D, Aggarwal A (2015) Lorazepam: a weapon of offence. J Clin Diagn Res 9:HD01-HD02

Soltaninejad K, Hassanian-Moghaddam H, Shadnia S (2014) Methadone related poisoning on the rise in Tehran, Iran. Asia Pac J Med Toxicol 3:104-109

Tiemensma M, Davies B (2018) Investigating drug-facilitated sexual assault at a dedicated forensic centre in Cape Town, South Africa. Forensic Sci Int 288: 115-122 Uddin MJ, Shahed FH, Bhowmik SK, Rashid R, Ghose A, Rahman 
MR (2002) Transport related poisoning - an untapped public health problem. The Healer 9:40-42

Wang X, Johansen SS, Nielsen MKK, Linnet K (2018) Hair analysis in toxicological investigation of drug-facilitated crimes in Denmark over a 8-year period. Forensic Sci Int 285:e1-e12

Zerbo S, Milone L, Scalici E, Procaccianti S, Nardello R, Spagnolo EV, Piscionieri D, Argo A (2018) Medico legal procedures related to sexual assault: a 10-year retrospective experience of a Daphne protocol application. Egypt J Forensic Sci 8:4

Submit your manuscript to a SpringerOpen ${ }^{\circ}$ journal and benefit from:

- Convenient online submission

- Rigorous peer review

- Open access: articles freely available online

- High visibility within the field

- Retaining the copyright to your article

Submit your next manuscript at $\boldsymbol{\nabla}$ springeropen.com 